\author{
Manuel Eduardo Loaiza Fernandez
}

Calibração de Múltiplas Câmeras baseado em um Padrão Invariante

Tese apresentada como requisito parcial para obtenção do título de Doutor pelo Programa de PósGraduação em Informática do Departamento de Informática da PUC-Rio.

Orientadores: Marcelo Gattass Co-Orientador: Alberto Barbosa Raposo 
Pontifícia Universidade Catálica $_{\text {Do Rio de Janelro }}$

Manuel Eduardo Loaiza Fernandez

\section{Calibração de Múltiplas Câmeras baseado em um Padrão \\ Invariante}

Tese apresentada como requisito parcial para obtenção do título de Doutor pelo Programa de Pós-Graduação em Informática do Departamento de Informática do Centro Técnico Científico da PUC-Rio. Aprovada pela Comissão Examinadora abaixo assinada.

Prof. Marcelo Gattass

Orientador

Departamento de Informática PUC-Rio

Prof. Alberto Barbosa Raposo

Co-Orientador

Departamento de Informática PUC-Rio

Prof. Waldemar Celes Filho Departamento de Informática PUC-Rio

Prof. Raul Queiroz Feitosa Departamento de Engenharia Elétrica PUC-Rio

Prof. Paulo Cezar Pinto Carvalho Instituto Nacional de Matemática Pura e Aplicada IMPA

Prof. Romero Tori Escola Politécnica da Universidade de São Paulo USP

Prof. José Eugenio Leal Coordenador Setorial do Centro Técnico Científico - PUC-Rio

Rio de Janeiro, 20 de agosto de 2009 
Todos os direitos reservados. É proibida a reprodução total ou parcial do trabalho sem autorização da universidade, do autor e do orientador.

\section{Manuel Eduardo Loaiza Fernandez}

Graduou-se em Engenharia de Sistemas na Faculdade de Produção e Serviços da Universidad Nacional de San Agustín (UNSA), Arequipa - Perú em 2002 e mestrado em Informática pela Pontifícia Universidade Católica do Rio de Janeiro (PUC-Rio), Rio de Janiero -Brasil em 2005. Atualmente pertence ao Grupo de Computação Gráfica Tecgraf da Pontifícia Universidade Católica do Rio de Janeiro. Tem experiência na área de Ciência da Computação, com ênfase em Computção Gráfica, Visualização 3D, Realidade Virtual, Realidade Aumentada e Visão Computacional.

Ficha Catalográfica

Loaiza Fernandez, Manuel Eduardo

Calibração de múltiplas câmeras baseado em um padrão invariante / Manuel Eduardo Loaiza Fernandez ; orientador: Marcelo Gattass ; co-orientador: Alberto Barbosa Raposo. - 2009.

131 f. : il. (color.) ; $30 \mathrm{~cm}$

Tese (Doutorado em Informática)-Pontifícia Universidade Católica do Rio de Janeiro, Rio de Janeiro, 2009.

Incluí referências bibliográficas.

1. Informática - Teses. 2. Calibração de câmera. 3. Geometria epipolar. 4. Estrutura a partir do movimento. 5. Características projetivas invariante. 6. Processamento de imagem. 7. Rastreamento óptico. I. Gattass, Marcelo. II. Raposo, Alberto Barbosa. III. Pontifícia Universidade Católica do Rio de Janeiro. Departamento de Informática. IV. Título. 


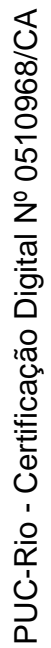

À minha esposa, Mayra, ao meu filho, Diego e à minha família. 


\section{Agradecimentos}

A Deus, por ter me dado a vida e a força necessária para continuar cada dia com o mesmo entusiasmo e dedicação.

A minha esposa, Mayra e meu filho Diego, pela paciência tida neste últimos anos.

Aos meus pais, Eduardo e Violeta e meus avós, Clotilde, Edgar e Blanca, pelo amor e exemplo de vida que me deram e que sempre lembro em todos os momentos da minha vida.

Aos meus tios Oscar, Milar, Enrique, Cira, Frida, Marlene, Gloria, Digber e Gladis pelos conselhos e ajuda que recebi deles.

Aos meus irmãos, Luis e Marizze, por todo o carinho e ânimos que me deram durante estes dois anos.

Aos meus orientadores Marcelo Gattass e Alberto Barbosa Raposo, pela confiança depositada no meu trabalho e por todos os ensinamentos repassados ao longo dos anos. Agradeço-lhes profundamente por seu apoio e amizade.

Aos meus amigos e colegas do Tecgraf Felipe, Peter Hohl, Lucas, Luciano, Cesar, Cristina, Gustavo por terem me presenteado com suas amizades e apoio durante o desenvolvimento desta tese.

E finalmente, a todas as pessoas que não foram mencionadas, mas que foram importantes em algum momento da minha vida.

Ao CNPq e à PUC-Rio, pelos auxílios concedidos, sem os quais este trabalho não poderia ter sido realizado. 


\section{Resumo}

Loaiza Fernandez, Manuel Eduardo; Gattass, Marcelo; Raposo, Alberto B. Calibração de Múltiplas Câmeras baseado em um Padrão Invariante. Rio de Janeiro, 2009. 131p. Tese de Doutorado - Departamento de Informática, Pontifícia Universidade Católica do Rio de Janeiro.

O processo de calibração de câmeras é uma etapa importante na instalação dos sistemas de rastreamento óptico. Da qualidade da calibração deriva o funcionamento correto e preciso do sistema de rastreamento. Diversos métodos de calibração têm sido propostos na literatura em conjunto com o uso de artefatos sintéticos definidos como padrões de calibração. Esses padrões, de forma e tamanho conhecidos, permitem a aquisição de pontos de referência que são utilizados para a determinação dos parâmetros das câmeras. Para minimizar erros, esta aquisição deve ser feita em todo o espaço de rastreamento. A fácil identificação dos pontos de referência torna o processo de aquisição eficiente. A quantidade e a qualidade das relações geométricas das feições do padrão influenciam diretamente na precisão dos parâmetros de calibração obtidos. É nesse contexto que esta tese se encaixa, propondo um novo método para múltipla calibração de câmeras, que é eficiente e produz resultados tão ou mais precisos que os métodos atualmente disponíveis na literatura. Nosso método também propõe um novo tipo de padrão de calibração que torna a tarefa de captura e reconhecimento de pontos de calibração mais robusta e eficiente. Deste padrão também derivam relações que aumentam a precisão do rastreamento. Nesta tese o processo de calibração de múltiplas câmeras é revisitado e estruturado de forma a permitir uma comparação das principais propostas da literatura com o método proposto. Esta estruturação também dá suporte a uma implementação flexível que permite a reprodução numérica de diferentes propostas. Finalmente, este trabalho apresenta resultados numéricos que permitem tirar algumas conclusões.

\section{Palavras-chave}

Calibração de câmera; Geometria epipolar; Estrutura a partir do movimento; Características projetivas invariante; Processamento de imagem; Rastreamento óptico. 


\section{Abstract}

Manuel Eduardo Loaiza Fernandez; Gattass, Marcelo (Advisor); Raposo, Alberto B. (Co-Advisor). Multiple Camera Calibration Based on Invariant Pattern. Rio de Janeiro, 2009. 131p. DSc. Thesis Departamento de Informática, Pontifícia Universidade Católica do Rio de Janeiro.

The calibration of multiple cameras is an important step in the installation of optical tracking systems. The accuracy of a tracking system is directly related to the quality of the calibration process. Several calibration methods have been proposed in the literature in conjunction with the use of artifacts, called calibration patterns. These patterns, with shape and size known, allow the capture of reference points to compute camera parameters. To yield good results these points must be uniformly distributed over the tracking area. The determination of the reference points in the image is an expensive process prone to errors. The use of a good calibration pattern can reduce these problems. This thesis proposes a new multiple camera calibration method that is efficient and yields better results than previously proposed methods available in the literature. Our method also proposes the use of a new simple calibration pattern based on perspective invariant properties and useful geometric properties. This pattern yields robust reference point identification and more precise tracking. This thesis also revisits the multiple calibration process and suggests a framework to compare the existing methods including the one proposed here. This framework is used to produce a flexible implementation that allows a numerical evaluation that demonstrates the benefits of the proposed method. Finally the thesis presents some conclusions and suggestions for further work.

\section{Keywords}

Camera Calibration; Epipolar Geometry; Structure from motion; Projective invariants; Image processing; Optical tracking. 


\section{Sumário}

Notações

1 Introdução 16

$\begin{array}{ll}\text { 1.1. Motivação } & 17\end{array}$

$\begin{array}{ll}\text { 1.2. Objetivo } & 19\end{array}$

1.3. Contribuições 20

$\begin{array}{ll}\text { 1.4. Organização da tese } & 21\end{array}$

2 Múltipla calibração de câmeras $\quad 23$

2.1. Primeira etapa: aquisição de dados para calibração 24

2.1.1. Padrões usados na calibração de câmeras 26

2.1.2. Padrão planar - 2D 27

2.1.3. Padrão unidimensional - 1D 29

2.1.4. Padrão adimensional 32

2.2. Segunda etapa: calibração inicial 35

2.2.1. Calibração fotogramétrica 35

2.2.1.1. Calibração baseada no cálculo de homografias 36

2.2.1.2. Cálculo da homografia 36

2.2.1.3. Determinação dos parâmetros intrínsecos e extrínsecos 38

2.2.1.4. Determinação dos coeficientes de distorção da lente 41

2.2.2. Auto-calibração 43

2.2.2.1. Geometria epipolar 44

2.2.2.2. Matriz fundamental 46

2.2.2.3. Cálculo da matriz fundamental 48

2.2.2.4. Determinação dos parâmetros extrínsecos 50

2.3. Terceira etapa: otimização da calibração 52

2.3.1. Funções de controle 53

2.3.1.1. Reprojeção 3D - 2D 53

2.3.1.2. Reprojeção 2D - 2D 54

2.3.1.3. Distância 3D entre pontos de referência reconstruídos 55 
2.4. Distribuição das câmeras $\quad 57$

2.4.1. Distribuição centralizada de câmeras 57

2.4.2. Distribuição descentralizada das câmeras 58

3 Trabalhos relacionados $\quad 61$

3.1. Método proposto por Jean-Yves Bouguet 62

3.2. Método proposto por Borghese 64

$\begin{array}{ll}\text { 3.3. Método proposto por Svoboda } & 67\end{array}$

3.4. Método proposto por Kurillo 69

4 Método proposto 72

4.1. Descrição do método

4.1.1. Aquisição de dados $\quad 77$

4.1.1.1. Captura do padrão planar 77

$\begin{array}{ll}\text { 4.1.1.2. Captura do padrão invariante } & 78\end{array}$

4.1.1.2.1. Treinamento do padrão invariante 79

4.1.1.2.2. Captura da amostra do padrão invariante 83

4.1.2. Calibração inicial 85

4.1.2.1. Calibração fotogramétrica 85

4.1.2.2. Auto-calibração 86

4.1.2.3. Otimização dos parâmetros 87

4.1.2.3.1. Definição da função de erro global 88

4.1.2.3.2. Colinearidade 2D e 3D 89

4.2. Sumarizando o método 90

$\begin{array}{ll}5 \text { Resultados } & 97\end{array}$

5.1. Equipamento e ambiente de teste 98

5.2. Metodologia dos testes 101

5.3. Descrição dos resultados 105

6 Conclusões 111

6.1. Trabalhos futuros 112

7 Referências Bibliográficas $\quad 114$ 
8 Apêndice A: Modelo de câmera 119

8.1.1. Sistemas de coordenadas 119

8.1.2. Transformações 120

8.1.3. Parâmetros extrínsecos 121

8.1.4. Parâmetros intrínsecos 122

8.1.5. Coeficientes de distorção das lentes 126

9 Apêndice B: Projetivas Invariantes 129

9.1. Calculo do $P^{2}$-Invariant para 4 pontos colineares 130 


\section{Lista de figuras}

Figura 1: Aplicações de realidade virtual e aumentada. 16

Figura 2: Sistemas de rastreamento óptico. 18

Figura 3: Sistema estéreo e de múltiplas câmeras. 23

Figura 4: Padrões planares usados para calibração de câmera

em trabalhos como, (a) Bouguet [5], (b) Zhang [40] e

Tsai [35][36] e (c) Heikkila [11].

27

Figura 5: Padrões planares usados em (a) Olsen e

Hoover [25] e (b) Bouguet [5]. 28

Figura 6: Padrão de calibração e captura proposto no trabalho de Borghese[4].

Figura 7: (a) Ambiente com uso de marcadores

retrorreflexivos em Uematsu et al.[37], (b) marcadores

coloridos usados como padrão em Mitchelson et al.[22].

Figura 8: Marcadores adimensionais propostos por

(a)Svoboda et al. [32], (b)Pintaric et al. [28] e [13].

Figura 9: Elementos da geometria epipolar - linha epipolar, epipolos, linha base, plano epipolar.

Figura 10: Correspondência entre duas vistas de um sistema estéreo de câmeras.

Figura 11: Ambiguidade da definição das quatro possíveis soluções para $R$ e $t[10]$.

Figura 12: Exemplificação do erro definido pela distância

da linha epipolar ao ponto correspondente.

Figura 13: Distribuição centralizada de câmeras. 58

Figura 14: Distribuição descentralizada de câmeras.

Figura 15: Calibração de um sistema estéreo de câmeras feita por Bouguet [5].

62

Figura 16: Padrões de calibração usados em [1][4][39]

Figura 17: O ponteiro (a), distribuição de 16 câmeras (b) e esquema da múltipla calibração de câmeras definido por Svoboda et al.[32] 
Figura 18: Análise da correlação de um sistema composto por cinco câmeras usando teoria de grafos definida em [14].

Figura 19: Evolução dos padrões (a) planar, (b) unidimensional,

(c) adimensional.

Figura 20: Padrões invariantes e processamento 2D para identificação individual.

Figura 21: Captura do padrão planar para calibração fotogramétrica. $\quad 77$

Figura 22: Processo de treinamento feito simultaneamente num sistema de 4 câmeras.

Figura 23: Identificação grupal e etiquetação individual dos marcadores que compõem os padrões invariantes.

Figura 24: Captura simultânea dois padrões invariantes num sistema de quatro câmeras.

Figura 25: Fluxograma geral do método de múltipla calibração de câmeras proposto nesta tese. $\quad 91$

Figura 26: Fluxograma do processo de calibração fotogramétrica.

Figura 27: Fluxograma do processo de treinamento dos padrões invariantes.

Figura 28: Fluxograma do processo de captura dos padrões invariantes previamente treinados.

Figura 29: Fluxograma do processo de captura dos padrões invariantes previamente treinados (Continuação).

Figura 30: Fluxograma do processo de auto - calibração.

Figura 31: Fluxograma do processo de otimização final dos parâmetros das câmeras estimados na calibração inicial.

Figura 32: (a) Câmeras web Logitech, (b) e (c) Câmeras Fire-l.

Figura 33: Três sistemas estéreo de câmeras formados pelas seis câmeras usadas para os testes.

Figura 34: (a) Sistema X e (b) sistema $Y$ de quatro câmeras. 99

Figura 35: Dois padrões invariantes usados nos testes. 100

Figura 36: Duas câmeras firewire conectadas em série. 100

Figura A1: Modelo de captura da câmera Pinhole [17].

Figura A2: Parâmetros extrínsecos na modelagem da 
transformação entre os SCM para o SCC [17].

Figura A3: Parâmetros intrínsecos na modelagem da transformação entre o SCC para o SCI [17].

Figura A4: Projeção de um ponto $P C$ no SCC para um ponto $P^{\prime}$ no SCl [17].

Figura A5:Transformação do SCI para o SCP[17].

Figura A6: Distorção causada por distintos tipos de lente -

(a) Barrel,(b) Pincushion. 


\section{Lista de tabelas}

Tabela 1: Valores dos identificadores para cada padrão invariante usado nos testes.

Tabela 2: Resultados do erro médio medidos na amostra $B, 1000$ vistas, capturada para teste da calibração realizada do sistema $\mathbf{X}$ de quatro câmeras firewire.

Tabela 3: Resultados do erro médio medidos na amostra $B, 1000$ vistas, capturada para teste da calibração realizada do sistema $\mathbf{Y}$ de quatro câmeras, duas firewire e duas usb.

Tabela 4: Ganho percentual ao analisar os resultados apresentados na tabela 1.

Tabela 5: Ganho percentual ao analisar os resultados apresentados na tabela 2.

Tabela 6: Comparação valor do erro médio referente da medição da distância entre marcadores definidos em métodos acadêmicos e produtos comerciais. 


\section{Notações}

Para melhorar a leitura e interpretação das variáveis usadas para descrever algumas fórmulas apresentadas dentro dos capítulos desta tese, a seguir serão apresentadas algumas descrições básicas destas notações.

- K é a matriz que contem a informação dos parâmetros intrínsecos de uma câmera (matriz de dimensão $3 \times 3$ ).

- P é a matriz de projeção da câmera (matriz de dimensão $3 \times 4$ )

- $\mathbf{r}_{1}, \mathbf{r}_{2}, \mathbf{r}_{3}$ são os vetores unitários que compõem uma matriz de rotação $\mathrm{R}$ (vetores de dimensão 3).

- $\mathbf{R}$ é a matriz de rotação (matriz de dimensão $3 \times 3$ ).

- $\quad \mathbf{T}, \boldsymbol{t}$ são representações do vetor translação (vetor de dimensão 3).

- $[\mathbf{u} \mathbf{v}]^{\mathbf{T}}$ é o vetor que representa um ponto expresso em coordenadas da imagem.

- $\left[\begin{array}{llll}\mathbf{X w} & \mathbf{Y w} & \mathbf{Z w}\end{array}\right]^{\mathrm{T}}$ é o vetor que representa um ponto expresso em coordenadas do mundo.

- H é uma matriz de homografia e $\mathrm{h}_{\mathrm{ij}}$ são os componentes na fila $\mathrm{i}$ coluna j dessa matriz (matriz de dimensão $3 \times 3$ ).

- $\left(\mathbf{u}_{\mathbf{d}}, \mathbf{v}_{\mathbf{d}}\right)$ coordenadas de um ponto na imagem onde esta presente a distorção gerada pela lente da câmera.

- $\left(\mathbf{u}_{\mathbf{u}}, \mathbf{v}_{\mathbf{u}}\right)$ coordenadas de um ponto na imagem onde foi corrigida distorção gerada pela lente da câmera.

- $\overrightarrow{A B}$ vetor definido entre os pontos $\mathrm{A}$ e $\mathrm{B}$.

- F é a matriz fundamental (matriz de dimensão $3 \times 3$ ).

- $\mathbf{E}$ é a matriz essencial (matriz de dimensão $3 \times 3$ ).

- $\quad \mathcal{E}$ é uma variável que representa um valor de erro.

- M é um ponto no mundo expresso em coordenadas homogêneas (vetor de dimensão 4 ).

- m é um ponto na imagem expresso em coordenadas homogêneas (vetor de dimensão 3 ). 